\title{
Three-dimensional Analysis of Mixed Convection in a Differentially Heated Lid-driven Cubic Enclosure
}

\author{
Nasreddine Benkacem*, Nader Ben Cheikh and Brahim Ben Beya \\ Department of Physics, Faculty of sciences, Tunis ELMANAR University, Campus Universities, 2092 El-Manar II, Tunis, Tunisia
}

\begin{abstract}
To study the intlicate three-dimensional flow structures and the companion heat transfer rates in a differentially heated lid-driven cubic cavity, a numerical methodology based on the finite volume method and a full multigrid acceleration is utilized in this note. The four remaining walls fowling the cubic cavity are adiabatic. The working fluid is air so the Prandtl number equates to 031. Numerical solutions are generated for representative combinations of the controlling Reynolds number inside $100<R e<1000$ and the Richardson number inside $0.001<R i<10$. Typical sets of streamlines and isotherms are presented to analyze the tortuous circulatory flow patterns set up by the competition between the forced flow created by the moving wall and the buoyancy force of the fluid. Correlations between the average Nusselt number through the cold wall and the Richardson number were established for the mentioned Reynolds numbers.
\end{abstract}

Keywords: Three-dimensional analysis; Lid-driven cubic cavity; Mixed convection; Numerical simulation; Multigrid method

\section{Introduction}

The problem on laminar mixed convection in cavities has multiple applications in the field of thermal engineering. Such problems are of great interest, for example in electronic device cooling, highperformance building insulation, multi shield structures used for nuclear reactors, food processing, glass production, solar power collector, etc. Numerous studies on lid-driven cavity flow and heat transfer involving different cavity configurations, various fluids and imposed temperature gradients have been continually published in the literature.

The numerical simulations of Moallemi and Jang [1] focused on two-dimensional laminar flow induced by Reynolds number 100 $\leq \operatorname{Re} \leq 1000$, and small-to-moderate Prandtl number $0.01 \leq \operatorname{Pr} \leq 50$ on the flow and heat transfer features in a cavity for different levels of the Richardson numbers. These authors found that the influence of buoyancy on the flow and heat transfer are to be more pronounced for higher values of $\mathrm{Pr}$, if Re and Gr are kept constant.

Sharif [2] performed a numerical investigation with flow visualization of laminar mixed convective heat transfer in twodimensional shallow rectangular driven cavities of aspect ratio 10 . The top moving plate of the cavity is set at a higher temperature than the bottom stationary plate. The fluid Prandtl number is taken as 6 , representative of water. The effects of inclination of such a cavity on the flow and thermal fields were also investigated for inclination angles ranging from $0^{\circ}$ to $30^{\circ}$. It was concluded that the average or overall Nusselt number increases mildly with cavity inclination for the dominant forced convection case dictated by $\mathrm{Ri}=0.1$. In contrast, it increases much more rapidly with inclination for the other dominant natural convection case dictated by $\mathrm{Ri}=100$.

Prasad et al. [3] numerically studied mixed convection inside a rectangular cavity where the two vertical walls are maintained at cold temperature. In one case, the top-moving wall is maintained at hot temperature and the bottom is at a cold temperature and in the other case, the top is at a cold temperature and the bottom is at a hot temperature. They concluded that when the negative is increased, a strong convection is manifested for aspect ratios equal to 0.5 and 1.0. Even more, a Hopf bifurcation occurs at for the aspect ratio 2.
Mohammad and Viskanta [4] numerically examined two and three-dimensional laminar flow and heat transfer in a Rayleigh-Bénard container. They established that the lid motion annihilates all forms of convective cells due to heating from below for finite size cavities. Aydin et al. [5] conducted a numerical investigation to analyze the transport mechanism of mixed convection in a shear and buoyancy-driven cavity having a locally heated lower wall and moving cooled sidewalls. In addition, other numerical studies such as Han and Kuehn [6] and Oztop and Dagtekin [7] were carried out on this topic.

Iwatsu et al. [8] performed a numerical investigation on the effect of external excitation on the flow structure in a square cavity. The results have shown similar flow structure to steady driven-cavity flows when utilizing small frequency values. Such a similarity, however, vanished when large frequency values were implemented. A subsequent work by Iwatsu et al. [9] carried out a numerical study of the viscous flow in a heated driven-cavity under thermal stratification, where the oscillating lid was maintained at a temperature higher than the lower wall. Their collection of results had revealed significant augmentation in heat transfer rate at particular lid frequency values, which convincingly indicates the existence of the resonance phenomena.

A detailed literature survey reveals that the majority of existing numerical investigations are restricted to two dimensional configurations. In this vein, 2D models are deficient because they do not always realistically capture the intricacies inherent to the flow behaviour. Because of these shortcomings, 3D models have to be undertaken to guarantee accuracy. A limited number of articles falls into this general category and has been reported in the literature.

*Corresponding author: Nasreddine Benkacem, Department of Physics, Faculty of sciences, Tunis ELMANAR University, Campus Universities, 2092 El-Manar II, Tunis, Tunisia, Tel: +71873 366; E-mail: nasreddine.benkacem@gmail.com

Received February 05, 2014; Accepted March 26, 2015; Published March 31, 2015

Citation: Benkacem N, Cheikh NB, Beya BB (2015) Three-dimensional Analysis of Mixed Convection in a Differentially Heated Lid-driven Cubic Enclosure. J Appl Mech Eng 4: 159. doi:10.4172/2168-9873.1000159

Copyright: (c) 2015 Benkacem N, et al. This is an open-access article distributed under the terms of the Creative Commons Attribution License, which permits unrestricted use, distribution, and reproduction in any medium, provided the original author and source are credited. 
Among others, Iwatsu [10] numerically studied three dimensional mixed convective flows in a cubical container with a steady vertical temperature stratification. He observed that the three dimensional effects are intensified as Re increases. Mohammad and Viskanta [11] conducted three-dimensional numerical simulation of mixed convection in a shallow driven cavity heated from the top moving wall and cooled from below. The cavity was filled with a stably stratified fluid encompassing a relative large range of Rayleigh and Richardson numbers. In a consecutive number of papers, Freitas et al. [12] and Freitas and Street [13] carried out a numerical study of the viscous flow in a rectangular cavity of depth-to-spanwise aspect ratio 3 at. They discovered the existence of meridional vortices and considerable flow unsteadiness.

In view of the foregoing statements, it seems that the problem of three dimensional laminar mixed convection heat transfers in a differentially heated lid-driven cubic cavity has not been addressed yet. In this paper, we undertake this task varying the Reynolds number in the Re-interval and the Richardson number in the Ri-interval for air $(\operatorname{Pr}=0.71)$ as the working fluid. The transport processes will be investigated with the finite volume method and the discussion will revolve around the precise determination of steady velocity and temperature fields. In addition, the average Nusselt number will be documented for all cases studied.

The paper is organized as follows: in the second section the physical system is formulated; the numerical methodology is briefly described in the third section and subsequently validated. The computed results are presented and discussed in the fourth section. In the final section, the most important findings of this study are summarized.

\section{Physical System}

The physical system under study is sketched in Figure 1. It basically consists of a cubic cavity with side filled with air. The applicable flow and temperature boundary conditions are described next. The top lid imparts a steady sliding motion with a uniform velocity, while the other walls are stationary. The cavity is differentially heated over the vertical sides. The left hot wall has a temperature and the right cold wall has a temperature where in. In addition, the remaining walls are considered adiabatic.

\section{Numerical Methodology and Algorithm Validation}

The governing equations for unsteady, incompressible laminar flow consist of the continuity equation, the Navier-Stokes equations accounting for the Boussinesq approximation and the energy equation. The non-dimensional equations are collectively written in tensor notation as follows:

Continuity equation:

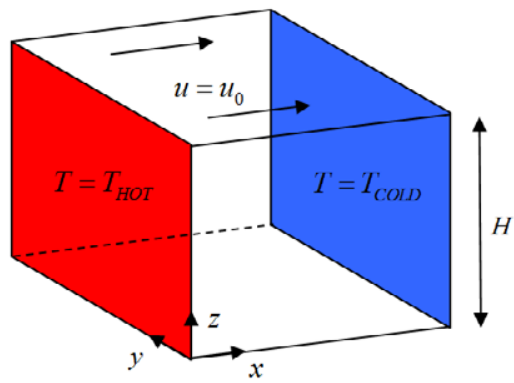

Figure 1: Physical system and Cartesian coordinates.

$$
\frac{\partial u_{i}}{\partial x_{i}}=0,
$$

Three Momentum equations:

$$
\frac{\partial u_{i}}{\partial t}+\frac{\partial\left(u_{i} u_{j}\right)}{\partial x_{j}}=-\frac{\partial p}{\partial x_{i}}+\frac{1}{\operatorname{Re}}\left(\frac{\partial^{2} u_{i}}{\partial x_{i} \partial x_{i}}\right)+R i \theta \delta_{i 3},
$$

Energy equation:

$$
\frac{\partial \theta}{\partial t}+\frac{\partial\left(u_{i} \theta\right)}{\partial x_{i}}=\frac{1}{R a \operatorname{Pr}}\left(\frac{\partial^{2} \theta}{\partial x_{i} \partial x_{i}}\right)
$$

Where $\mathrm{u}_{\mathrm{i}}=(\mathrm{u} ; \mathrm{v} ; \mathrm{w})$ the velocity components, pare is the kinematic pressure, and $\theta$ is the temperature, $\rho$ is the mass density, and $g$ is the gravitational acceleration. In Eq. (2), the symbol $\delta_{i j}$ stands for the Krönecker delta. The chosen scales in Equations (1)-(3) are the side $H$, the velocity $u_{0}=\sqrt{g \beta H \Delta T}$, the time $\mathrm{t}_{0}=\mathrm{H} / \mathrm{u}_{0}$ and the pressure $p_{0}=\rho u_{0}^{2}$. Further, the non-dimensional temperature is defined by $\beta$ $\theta=\frac{T-T_{R}}{T_{H O T}-T_{C O L D}}$, where the reference temperature $T_{R}=\frac{T_{H O T}+T_{C O L D}}{2}$ and the temperature scale is the lid-to-lid temperature difference $\mathrm{T}_{\text {нот }}-$ $\mathrm{T}_{\text {COLD }}$.

As presented above, the forced-natural convection problem is characterized by three non-dimensional parameters:

1) the Reynolds number $\operatorname{Re}=\frac{u_{0} H}{v}$ where $u_{o}$ is the impressed lid velocity;

2) The Prandtl number $\operatorname{Pr}=\frac{v}{\alpha}$, where $v$ is the kinematic viscosity, $\alpha$ the thermal diffusivity of the fluid;

3) The Grashof number $G r=\frac{\beta g \Delta T H^{3}}{v^{2}}$ in which $\beta$ is the coefficient of thermal expansion of the fluid, $g$ the gravity and $\Delta T=T_{\text {Hот }}-T_{\text {СоI }}$ the temperature difference between the hot and cold horizontal walls. Alternatively, $\mathrm{Gr}$ and Re are adequately blended in the mixedconvection parameter called the Richardson number $R i=\frac{G r}{\mathrm{Re}^{2}}$.

The unsteady Navier-Stokes and energy equations are discretized by a second-order time stepping finite difference procedure. The procedure adopted here deserves a detailed explanation. First, the non-linear terms in Eqs. (2) are treated explicitly with a secondorder Adams-Bashforth scheme. Second, the convective terms in Eq. (3) are treated semi-implicitly. Third, the diffusion terms in Eqs. (2) and (3) are treated implicitly. In order to avoid the difficulty that the strong velocity-pressure coupling brings forward, we selected a projection method described in Peyret and Taylor [14] and Achdou and Guermond [15].

A finite-volume method is implemented to discretize the NavierStokes and energy equations (Patankar [16], F. Moukhalled and M. Darwish [17], Kobayachi and Pereira and Pereira [18]). The advective terms in Eqs. (2) are discretized using a QUICK third-order scheme whereas a second-order central differencing (Hayase, Humphrey and Greif [19]) is applied in Eq. (3). The discretized momentum and energy equations are solved employing the red and black successive over relaxation method (RBSOR) in Press et al. [20], while the Poisson pressure correction equation is solved utilizing a full multi-grid method (Hortmann et al. [21], Mesquita and de Lemos [22], Nobile [23]). If specific details about the computational methodology are needed, the reader is directed to Ben Cheikh et al. [24]. Finally, the convergence of the numerical 3D velocity field and the 3D temperature 
Citation: Benkacem N, Cheikh NB, Beya BB (2015) Three-dimensional Analysis of Mixed Convection in a Differentially Heated Lid-driven Cubic Enclosure. J Appl Mech Eng 4: 159. doi:10.4172/2168-9873.1000159

Page 3 of 5

field is established at each time step when all residuals are forced to stay below $10^{-6}$. To secure steady state conditions the following criterion has to be satisfied:

$$
\sum_{i, j, k}\left|\Phi_{i, j, k}^{m+1}-\Phi_{i, j, k}^{m}\right| \leq 10^{-5}
$$

Where the generic variable $\Phi$ represents the set of four variables $(\mathrm{u}, \mathrm{v}, \mathrm{w})$ or $\theta$. In the above inequality, the superscript $m$ indicates the iteration number and the subscript sequence $(\mathrm{i}, \mathrm{j}, \mathrm{k})$ represents the space coordinates $x, y, z$.

For enhanced accuracy, the present numerical model was checked against the published numerical solution of Tric [25]. The outcomes of the one-to-one comparisons are documented in Table 1 for the average Nusselt number predictions and maximum velocities. It is observed here that the present numerical computations match very closely those of [25].

A second comparison to those of Iwatsu [10] relatively to a 3D mixed convection was undertaken. As shown in Table 2, good agreements are evident with respect to the result reported by [10].

\section{Results and Discussion}

The computed mixed convection flow and temperature fields in the lid-driven cubic cavity are examined in this section. The numerical results are presented in terms of streamlines and isotherms. The Reynolds number Re is varied two orders of magnitude between 100 and 1000. In addition, the Richardson number Ri is varied four orders of magnitude between 0.001 and 10. The Prandtl number is set at $\mathrm{Pr}=0.71$. We ran computations for nine different pairs of Ri and Re; that is: $(\mathrm{Ri}, \mathrm{Re})=(10,100),(10,400),(10,1000),(1,100),(1,400),(1,1000)$, $(0.001,100),(0.001,400)$ and $(0.001,1000)$. In harmony with this, the implications of varying Ri and Re will be adequately highlighted.

A series of trial calculation were conducted with two different variable grid distributions, i.e., $48 \times 48 \times 48$ and $64 \times 64 \times 64$. For the moderate case dealing with $\mathrm{Re}=400$ and $\mathrm{Ri}=1.0$, minor differences of less than $0.25 \%$ were detected between the flow and temperature results produced by the grid $48 \times 48 \times 48$ and those by the grid $64 \times 64 \times 64$. Consequently, to optimize the grid distribution appropriately, the grid $48 \times 48 \times 48$ was deemed adequate to perform all numerical computations. For completeness, the two grids were built using a tangent hyperbolic formulation. The smallest space intervals chosen in the three coordinate directions are $\Delta x_{\min }=\Delta y_{\min }=\Delta z_{\min }=2.25 \times 10^{-3}$, and are localised near the moving and stationary walls to capture the growth of the flow and thermal boundary layers adjacent to them. The time step was set to $\Delta \mathrm{t}=0.01$ for all computations.

\begin{tabular}{|c|c|c|c|c|c|c|}
\hline & & $R R R R=1111^{44}$ & & & $R R R R=1111^{55}$ & \\
\hline & Tric [25] & $\begin{array}{l}\text { Pres. } \\
\text { Work }\end{array}$ & $\begin{array}{l}\text { Err } \\
\%\end{array}$ & $\begin{array}{l}\text { Tric } \\
{[25]}\end{array}$ & $\begin{array}{l}\text { Pres. } \\
\text { Work }\end{array}$ & $\begin{array}{l}\text { Err } \\
\%\end{array}$ \\
\hline Grille & 813 & $48^{3}$ & & 813 & 483 & \\
\hline$u u_{m m} R R_{m m}$ & 16.719 & 16.634 & -0.51 & 43.90 & 44.06 & 0.36 \\
\hline$v v_{m m} R R_{m m}$ & 2.156 & 2.136 & -0.93 & 9.69 & 9.55 & -0.15 \\
\hline $\boldsymbol{w} \boldsymbol{w}_{m R R m m}$ & 18.983 & 18.942 & -0.22 & 71.06 & 70.85 & -0.30 \\
\hline NNuum $_{m m m}$ & 2.250 & 2.247 & -0.13 & 4.612 & 4.605 & -0.15 \\
\hline$N_{N u u_{3333}}$ & 2.054 & 2.054 & 0 & 4.337 & 4.332 & -0.12 \\
\hline
\end{tabular}

Table 1: Comparison of the computed average Nusselt number predictions and maximum velocities.

\begin{tabular}{|l|c|c|c|c|c|c|}
\hline Re & \multicolumn{2}{|c|}{ Ri=0.001 } & \multicolumn{2}{c|}{ Ri=1.0 } & \multicolumn{2}{c|}{ Ri=10.0 } \\
\hline & Ref [10] & Pres. Work & Ref [10] & Pres. Work & Ref [10] & Pres. Work \\
\hline $\mathbf{1 0 0}$ & 1.82 & 1.836 & 1.33 & 1.348 & 1.08 & 1.092 \\
\hline $\mathbf{4 0 0}$ & 3.99 & 3.964 & 1.50 & 1.528 & 1.17 & 1.130 \\
\hline $\mathbf{1 0 0 0}$ & 7.03 & 7.284 & 1.80 & 1.856 & 1.37 & 1.143 \\
\hline \multicolumn{7}{|c|}{ Table 2: Comparison of our results with [10]. } \\
\hline
\end{tabular}

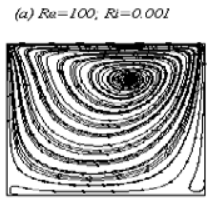

(d) $R=400, R=0.002$
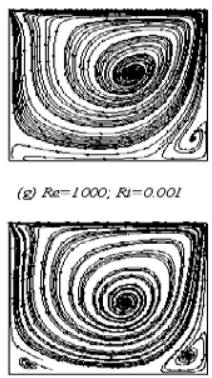

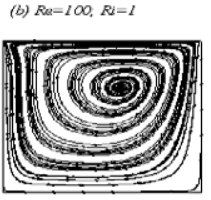

(e) $R e-400, R t=1$

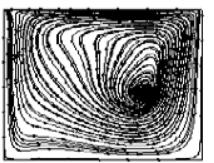

(h) $R e-1000 ; R t=1$

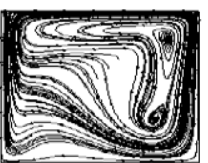

(c) $R e=100 ; R u=10$

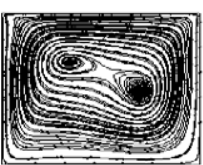

(t) $R e^{-400 ;}: R t=10$

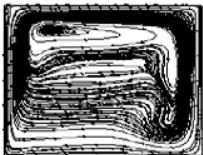

(a) $R=1000 ; \quad R=10$

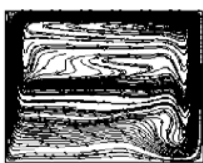

Figure 2: Trajectory particles at the $x-z$ mid-plane for different combinations of $R e$ and $R i$.

The mid-plane streamlines distributions for designated values of Re and $\mathrm{Ri}$ are displayed in Figure 2. We note that for the lowest Richardson number employed ( $\mathrm{Ri}=0.001)$, the trajectory of fluid particles is very similar to that corresponding to the classical lid-driven cavity [26] (Figure $2 \mathrm{a}-2 \mathrm{~d}-2 \mathrm{~g}$ ). Indeed, Figure $2 \mathrm{a}$ shows the flow structure in the cavity at $\mathrm{Re}=100$ with a primary vortex occupying the main part of the cavity. Two small recirculation cells are also emerging at the bottom corners as the Reynolds number goes through $\mathrm{Re}=400$ to $\mathrm{Re}=1000$.

When $\mathrm{Ri}$ is large $(\mathrm{Ri}=10)$, it is noticeable in $(\mathrm{Re}=100)$ the presence of two eddies localised in the proximity of the core region. With increments in Re, the right cell becomes feeble and amalgamates with the left one to provide only one stretched vortex. Interestingly, it is also noticed when $\operatorname{Re}=1000$, that the direction of the lid velocity causes the centre of the vortex to move from the left side to the right side as confirmed by Figure $2 \mathrm{i}$.

The case $(\mathrm{Ri}=1 ; \mathrm{Re}=100)$ is very similar to $(\mathrm{Ri}=0.001 ; \mathrm{Re}=100)$. In fact a primary cell is observed in the cavity with a little difference that its center is slightly moved downward. It is conspicuous in Figure $2 \mathrm{e}$ the effect of increasing the Reynolds number $(\mathrm{Re}=400)$ on the flow structure. The main vortex moves down and is somewhat dragged to the right side of the cold wall. For $\mathrm{Re}=1000$, the high lid velocity causes the division of the main vortex in two cells (see Figure $2 \mathrm{~h}$ ).

The qualitative features of the temperature field are demonstrated by plotting the perspective views of isotherms, as reflected in Figure 3. In fact, it is clearly discernible from the patterns of isotherms that, for the feeble value of Richardson number $(\mathrm{Ri}=0.001)$, the mechanically driven forced convection dominates the buoyancy-driven convection (Figure $3 \mathrm{a}, 3 \mathrm{~d}$ and $3 \mathrm{~g}$ ), implying that the forced convection is essentially due to the lid-movement. In contrast, as $\mathrm{Ri}$ increases to $\mathrm{Ri}=10$ the buoyant 


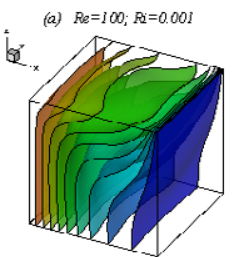

(a) $R e=400 ; R i=0.001$

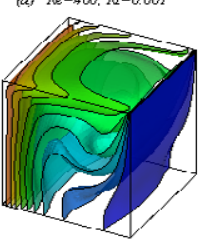

(g) $R_{e}=3000 ; R_{1}=0.001$

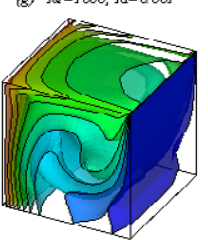

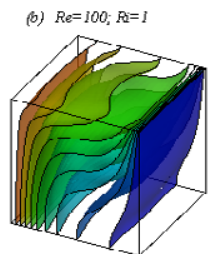

(e) $R e=400: R \mathrm{i}=1$

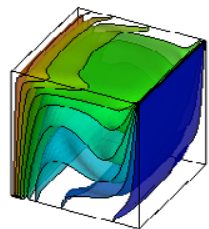

(h) $R_{e}=J 000 ; R_{i}=1$

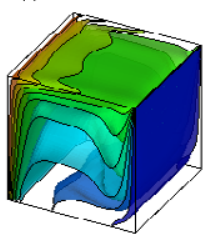

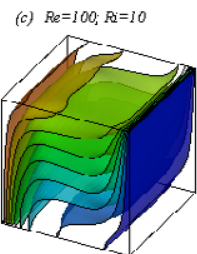

(e) $R e=400 ; R i=10$

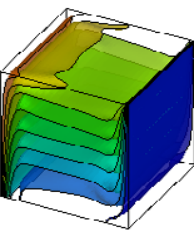

(i) $R_{e}=1000 ; P_{i}=30$

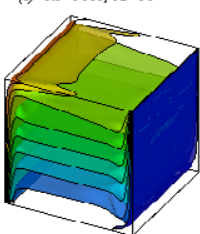

Figure 3: Isotherm plots for different combinations of $R e$ and $R i$.

\begin{tabular}{|l|c|c|c|}
\hline $\mathbf{R e}$ & $\mathbf{R i = 0 . 0 0 1}$ & $\boldsymbol{R i = 1 . 0}$ & $\boldsymbol{R i = 1 0 . 0}$ \\
\hline & $\begin{array}{c}(\text { NNNNh oooo; } \\
\text { NNNNccooccc })\end{array}$ & $\begin{array}{c}(\text { NNNNh oooo; } \\
\text { NNNNccoocccc })\end{array}$ & $\begin{array}{c}(\text { NNNNh oooo; } \\
\text { NNNNccoocccc })\end{array}$ \\
\hline $\mathbf{1 0 0}$ & $(2.1714 ; 2.1714)$ & $(2.6876 ; 2.6876)$ & $(4.3186 ; 4.3187)$ \\
\hline $\mathbf{4 0 0}$ & $(4.3276 ; 4.3285)$ & $(5.7232 ; 5.7241)$ & $(9.6408 ; 9.6420)$ \\
\hline $\mathbf{1 0 0 0}$ & $(6.6252 ; 6.6294)$ & $(9.3182 ; 9.3236)$ & $(15.8457 ; 15.852$ \\
\hline
\end{tabular}

Table 3: The average Nusselt number Nu at the cold and hot walls.

\begin{tabular}{|c|c|c|c|}
\hline $\boldsymbol{R e}$ & $\boldsymbol{a}$ & $\boldsymbol{b}$ & $\boldsymbol{\alpha} \boldsymbol{\alpha}$ \\
\hline $\mathbf{1 0 0}$ & 0.523 & 2.165 & 0.615 \\
\hline $\mathbf{4 0 0}$ & 1.421 & 4.303 & 0.575 \\
\hline $\mathbf{1 0 0 0}$ & 2.768 & 6.555 & 0.526 \\
\hline
\end{tabular}

Table 4: Values of coefficients $a a, b b a a b b c c \alpha \alpha$.

convection distorts the isotherm fields and three-dimensional patterns become more pronounced when Re increases (Figure 3c, 3f and 3i). The distortion of the isotherm field increases with Richardson number. In other words, the flow is principally dominated by buoyancy and the heat transfer is controlled mainly by natural convection, signifying that the forced convection due to the lid-movement is almost absent.

For $\mathrm{Ri}=1$, a compromise between the two phenomena, evoked previously, is clearly seen in Figures 2b, 2e, 2h, 3b, 3e and $3 \mathrm{~h}$.

In order to assess the average heat transfer distribution along the vertical walls, the Nusselt number is introduced and is defined by:

$N u=\int_{0}^{1} \int_{0}^{1}-\left.\frac{\partial \theta}{\partial x}\right|_{x=0 \text { or } x=1} d y d z$.

Table 3 lists the average Nusselt number $\mathrm{Nu}$ at the cold and hot walls for the computations obtained for the nine combinations (Re,Ri) studied. The results convincingly indicate that when $\operatorname{Re}$ is small $(\mathrm{Re}=100)$, the heat transfer through the cold and the hot walls exhibit similar trends for each value of Ri. For this same Reynolds number, the average Nusselt number increases with the Richardson number. By increasing the Reynolds number, values of Nusselt number increase and small differences between $\mathrm{Nu} u_{\text {hot }}$ and $\mathrm{Nu}$ cold are observed. When Ri is small at high values of Re, the difference between $N u_{\text {hot }}$ and $N u_{\text {cold }}$ augment.

Relatively to the heat transfer through the cold wall, a correlation between $\mathrm{Nu}$ and $\mathrm{Ri}$ was established. In fact, several computations (for each Reynolds number) demonstrate clearly the existence of a relation expressed as:. $N u=a \times R i^{\alpha}+b$. Table 4 lists the values of coefficients, $\mathrm{b}$ and $\alpha$.

\section{Conclusions}

The current investigation addressed three-dimensional laminar mixed convection in a lid-driven cubic cavity filled with air $(\operatorname{Pr}=0.71)$ for suitable combinations of three different Reynolds numbers and three different Richardson numbers. The effects of varying both Reynolds and Richardson numbers on the resulting convection are investigated. Interesting behaviours of the flow and thermal fields with varying Reynolds and Richardson numbers are observed.

When small $R \mathrm{i}$ is united with low $R \mathrm{e}$, a primary vortex is observed occupying the main part of the cavity and its intensity is slightly modified when Re increased. In addition, two minor secondary recirculating vortices are observed at the bottom corners as the Reynolds number goes through $R e=400$ to $R e=1000$. Furthermore, three dimensionalities of the isotherm patterns are manifested. In this case, the mechanically driven forced convection dominates the buoyancy-driven, implying that forced convection is essential due to the lid-movement.

When large $R \mathrm{i}$ is paired with low Re, two primary vortex are observed in the proximity of the core region and their intensity is considerably modified to provide only one stretched vortex when $R e$ increase. It is also seen that the buoyancy-driven dominates the mechanically driven forced convection.

The heat transfer characteristics inside the cubic cavity are improved significantly for low values of $R$ i due to the dominant effect of the mechanical effect provoked by the moving lid. The effects of both $R e$ and $R \mathrm{i}$ are also apparent in the values of the average Nusselt number. For high $R$ i united with large $R e$, the overall heat transfer and the convection mode dominates the picture. Finally for Reynolds number ranging from 100 to 1000 , a correlation between the averaged heat transfer $(\mathrm{Nu})$ and $\mathrm{Ri}$ has been reported.

\section{References}

1. Moallemi MK, Jang KS (1992) Prandtl number effects on laminar mixed convection heat transfer in a lid-driven cavity. Int $\mathrm{J}$ Heat Mass Transfer 35: 1881-1892.

2. Sharif MAR (2007) Laminar mixed convection in shallow inclined driven cavities with hot moving lid on top and cooled from bottom. Applied Thermal Engineering 27: 1036-1042.

3. Prasad YS, Das MK (2007) Hopf bifurcation in mixed flow inside a rectangular cavity. Int J Heat Mass Transfer 50: 3583-3598.

4. Mohammad AA, Viskanta R (1992) Laminar flow and heat transfer in RayleighBenard convection with shear Phys. Fluids A 4: 2131-2140.

5. Aydin O, Yang WJ (2000) Mixed convection in cavities with a locally heated lower wall and moving sidewalls, Numer. Heat Transfer, Part A: Applications 37: $695-710$

6. Han H, Kuehn TH (1989) A numerical simulation of double diffusive convection in a vertical rectangular enclosure. ASME HTD 107: 149-154.

7. Oztop HF, Dagtekin I (2004) Mixed convection in two sided lid driven 
Citation: Benkacem N, Cheikh NB, Beya BB (2015) Three-dimensional Analysis of Mixed Convection in a Differentially Heated Lid-driven Cubic Enclosure. J Appl Mech Eng 4: 159. doi:10.4172/2168-9873.1000159

differentially heated square cavity. Int J Heat Mass Transfer 47: 1761-11769.

8. Iwatsu R, Hyun JM, Kuwahara K (1992) Numerical simulation of flows driven by a torsionally oscillating lid in a square cavity. J Fluids Engineering 114: 143151.

9. Iwatsu R, Hyun JM, Kuwahara K (1992) Convection in a differentially-heated square cavity with a torsionally-oscillating lid. Int. J. Heat Mass Transfer 35 1069-1076.

10. Iwatsu R, Hyun JM (1995) Three-dimensional driven-cavity flows with a vertical temperature gradient. Int J Heat Mass Transfer 38: 3319-3328.

11. Mohammad AA, Viskanta $R$ (1995) Flow and heat transfer in a lid-driven cavity filled with a stably stratified fluid. Appl. Math. Modelling 19: 465-472.

12. Freitas CJ, Street RL, Findikakis AN, Koseff JR (1985) Numerical simulation of three-dimensional flow in a cavity. Int. J. Numer. Meth. Fluids 5: 561-575.

13. Freitas CJ, Street RL (1988) Non-linear transport phenomena in a complex recirculating flow: a numerical investigation. Int J Numer Meth Fluids 8: 769-802.

14. Peyret R, Taylor TD (1983) Methods for Fluid Flow. Springer-Verlag, Berlin, Germany.

15. Achdou Y, Guermond JL (2000) Convergence analysis of a finite element projection/Lagrange-Galerkin method for the incompressible Navier-Stokes equations. SIAM J Numer Anal 37: 799-826.

16. Patankar SV (1981) A calculation procedure for two-dimensional elliptic situations. Numer Heat Transfer 34: 409-425.

17. Moukhalled F, Darwish M (2000) A unified formulation of the segregated class of algorithm for fluid flow at all speeds. Numer. Heat Transfer, Part B: Fundamentals 37: 103-139.
18. Kobayachi MH, Pereira JMC, Pereira JCF (1999) A conservative finite-volume second-order-accurate projection method on hybrid unstructured grids. J Comput Phys 150: 40-75.

19. Hayase T, Humphrey JAC, Greif R (1992) A consistently formulated QUICK scheme for fast and stable convergence using finite-volume iterative calculation procedures. J Comput Phys 98: 108-118.

20. Press WH, Teukolsky SA, Vetterling WT, Flannery BP (1997) Numerica Recipes in Fortran 77: The Art of Scientific Computing. (second edition), Cambridge Press, London, UK.

21. Hortmann M, Peric M, Scheuerer G (1990) Finite volume multigrid prediction of laminar natural convection: benchmark solutions. Int $\mathrm{J}$ Numer Meth Fluids 11: 189-207.

22. Mesquita MS, De Lemos MGS (2004) Optimal multigrid solutions of dimensional convection-conduction problems. Appl Math Comput 152: 725-742.

23. Nobile $E$ (1996) Simulation of time-dependent flow in cavities with the correction multigrid method, Part I: mathematical formulation. Numer Heat Transf Part B Fundamentals 30: 341-350.

24. Cheikh NB, Beya BB, Lili T (2007) Benchmark solution for time-dependent natural convection flows with an accelerated full-multigrid method, Numerical Heat Transfer. Part B: Fundamentals 52: 131-151.

25. Tric E, Labrosse G, Betrouni M (2000) A first incursion into the 3D structure of natural convection of air in a differentially heated cubic cavity, from accurate numerical solutions. Int J Heat and Mass Transfer 43: 4043-4056.

26. Wong KL, Baker AJ (2002) A 3D incompressible Navier-Stokes velocityvorticity weakform 2nite element algorithm. Int J Numer Meth Fluids 38: 99-123. 